\title{
Ocular dimensions of the Chinese adolescents with keratoconus
}

\author{
Weijun Jian ${ }^{1,2 \dagger}$, Yang Shen ${ }^{1,2 \dagger}$, Yingjun Chen ${ }^{1,2}$, Mi Tian $^{1,2}$ and Xingtao Zhou ${ }^{1,2^{*}}$ (D)
}

\begin{abstract}
Background: Adolescent KC is a special segment of the general KC population because an adolescents's eyes are still susceptible to blurred vision and optical defocus during the sensitive period of ocular and visual development. In the present study, we evaluated the ocular dimensions of $53 \mathrm{KC}$ adolescents.

Methods: One hundred and six KC eyes of 53 (42 boys and 11 girls) KC adolescents (age $15.5 \pm 1.7$ years, range 11 to 18) were involved in this retrospective study. The eye with more severe KC (Severe Group) of each patient was compared with their less affected eye (Mild Group). Optical axial length (OAL) was measured by optical coherence biometry (IOL-master). Central corneal thickness, anterior chamber depth (ACD), flat keratometry value, steep keratometry value, and maximum keratometry value were assessed with an anterior segment analyzer (Pentacam HR). Anterior segment length (ASL) was manually measured from the 25 scheimpflug images captured by the Pentacam HR with the mean value recorded. The posterior segment length (PSL) was calculated with the formula "PSL = OAL-ASL".

Results: The mean ACD, OAL, ASL, and PSL values of the Severe Group were $3.51 \pm 0.32 \mathrm{~mm}, 24.76 \pm 1.24 \mathrm{~mm}$, $4.01 \pm 0.30 \mathrm{~mm}$ and $20.76 \pm 1.15 \mathrm{~mm}$. While those of the Mild Group were $3.36 \pm 0.29 \mathrm{~mm}, 24.97 \pm 1.40 \mathrm{~mm}$, $3.94 \pm 0.35 \mathrm{~mm}$ and $21.03 \pm 1.31 \mathrm{~mm}$. The Severe Group has significantly higher $A C D(t=4.539, P<0.001)$ value but lower OAL $(t=-3.120, P=0.003)$ and PSL $(t=-4.537, P<0.001)$ values when compared with those of the Mild Group. For the Severe Group, the Kmax values were significantly correlated with the SE values $(R=-0.385$, $P=0.004)$, the $A C D$ values $(R=0.375, P=0.006)$, the $A S L$ values $(R=0.308, P=0.025)$ and the PSL values $(R=-0.317$, $P=0.021)$, but not with the OAL values $(R=-0.220, P=0.114)$. In the Mild Group, the Kmax values were negatively correlated with the SE $(R=-0.577, P<0.001), \mathrm{OAL}(R=-0.533, P<0.001)$, and PSL $(R=-0.523, P<0.001)$ values, but not with $A C D(R=-0.110, P=0.434)$ or ASL $(R=-0.182, P=0.192)$ values.
\end{abstract}

Conclusions: For adolescent KC, the more keratoconic eyes may be characterized by deeper ACD but shorter OAL and PSL, when compared with the less affected ones.

Keywords: Child health (paediatrics), Cornea, Eye (globe), Optics and refraction, Anterior chamber

\section{Background}

Keratoconus (KC) is a chronic and degenerative corneal ectatic disease with an onset during early adulthood or even adolescence [1]. Generally, KC successively affects both eyes. With progressively thinning corneal thickness and steeping corneal curvature, $\mathrm{KC}$ would lead to refractive error (refractive myopia and irregular astigmatism) and impaired vision [2]. Nowadays, the improved

\footnotetext{
* Correspondence: doctzhouxingtao@163.com

${ }^{\dagger}$ Equal contributors

${ }^{1}$ The Eye and ENT Hospital of Fudan University, Shanghai, China

${ }^{2}$ The Key Lab of Myopia, Ministry of Health, People's Republic of China, 19 Baoging Road, Shanghai, Xuhui District, China
}

techniques of corneal topography and in-vivo corneal biomechanical analysis [3-8] significantly increase the ability to diagnose keratoconus. Therefore, it can be easier to detect $\mathrm{KC}$ cases in subclinical stages or in the adolescent population [9].

Adolescent $\mathrm{KC}$ is a special segment of the general $\mathrm{KC}$ population because an adolescents's eyes are still susceptible to blurred vision and optical defocus during the sensitive period of ocular and visual development [10-12]. However, recent $\mathrm{KC}$ studies have been mostly focused on investigating the structural changes in the anterior segment, including corneal shape (corneal curvature, antertior corneal 
elevation, posterior corneal elevation, corneal thickness), anterior chamber depth, anterior chamber angle [13-22]. The effect of $\mathrm{KC}$ on the ocular axial length in the adolescent population is poorly understood. In the present study, we evaluated the ocular dimensions of $53 \mathrm{KC}$ adolescents.

\section{Methods}

\section{Participants}

One hundred and six $\mathrm{KC}$ eyes of $53 \mathrm{KC}$ adolescents (42 boys and 11 girls) whose refractive error have been or have never been corrected by spectacles were recruited in this retrospective study from August 2015 to September 2016 at the Department of Ophthalmology of Eye and ENT hospital, Fudan University. The mean age was $15.5 \pm 1.7$ years (range 11 to 18 ). The eye with higher Kmax value (Severe Group) of each patient was compared with their lower Kmax value eye (Mild Group).

\section{Ophthalmologic examinations}

Each participant underwent manifest refractions (cycloplegic and non-cycloplegic) and best spectacles corrected distance acuity (BSCDVA) examinations. The anterior segmental parameters including flat keratometry value (K1), steep keratometry value (K2), maximum keratometry value (Kmax), central corneal thickness (CCT) and anterior chamber depth (ACD) were assessed with an anterior segment analyzer (Pentacam HR, Typ70900, Oculus Optikgeräte $\mathrm{GmbH}$, Wetzlar, Germany) at a sitting position. Images with an 'OK' in quality specification (QS) were analyzed. An optical coherence biometry (IOL-master 1322-734, Carl Zeiss Meditec AG, Jena, Germany) was applied to evaluate the optical axial length (OAL) value along the visual axis (line connecting the fixation point to the fovea, specifically from the anterior surface of the cornea to the retinal pigment epithelium layer of the fovea). Five measurements were continuously obtained from each eye at a sitting position, with the mean value of the five measurements calculated automatically.
The anterior segment length (ASL) of each eye (from the anterior surface of the cornea to the anterior capsule of the lens along the optical axis) was manually measured from the 25 scheimpflug images of each eye captured by the Pentacam HR, with the mean value recorded. The posterior segment length (PSL), which refers to the distance from the anterior capsule of the lens to the retinal pigment epithelium layer of the macula, was calculated with the formula "PSL = OAL-ASL" (Fig. 1).

\section{Data analysis and statistical evaluation}

Statistical analysis was performed using SPSS 19 (SPSS Inc., IBM). Normality check was conducted using the Kolmogorov-Smirnov Z test. All these parameters of the eyes in the Severe Group (with higher Kmax value) were compared with those of the eyes in the Mild Group. Paired-t test was used for the normal distribution data. If the data distribution was not normal, Wilcoxon signedrank test was employed for the analysis. The potential correlations between SE, Kmax and OAL were evaluated with Pearson correlation tests. Cut-off $P$ values were 0.05 .

\section{Results}

Refraction and the main topographic parameters

The mean manifest refraction (cycloplegic) spherical equivalent (SE) of the eyes in the Severe Group was $8.26 \pm 4.72$ diopters $(\mathrm{D})$, which was much more myopic $(P<0.05)$ than those in the Mild Group. $(-5.62 \pm$ 3.52D). As listed in Table 1 , the mean CCT value of the Severe Group was $459.3 \pm 45.8 \mu \mathrm{m}$, significantly lower $(\mathrm{t}=-7.933, P<0.001)$ than that of the Mild Group. $(504.3 \pm 39.9 \mu \mathrm{m})$ The mean values of K1, K2 and Kmax values of the Severe Group were $50.72 \pm$ $7.60 \mathrm{D}, 55.56 \pm 8.15 \mathrm{D}$ and $63.79 \pm 11.49 \mathrm{D}$, respectively. They were significantly higher than those of the Mild Group ( $\mathrm{t}=6.922, P<0.001 ; \mathrm{t}=8.614, P<0.001 ; \mathrm{t}=9.791$, $P<0.001$, respectively).

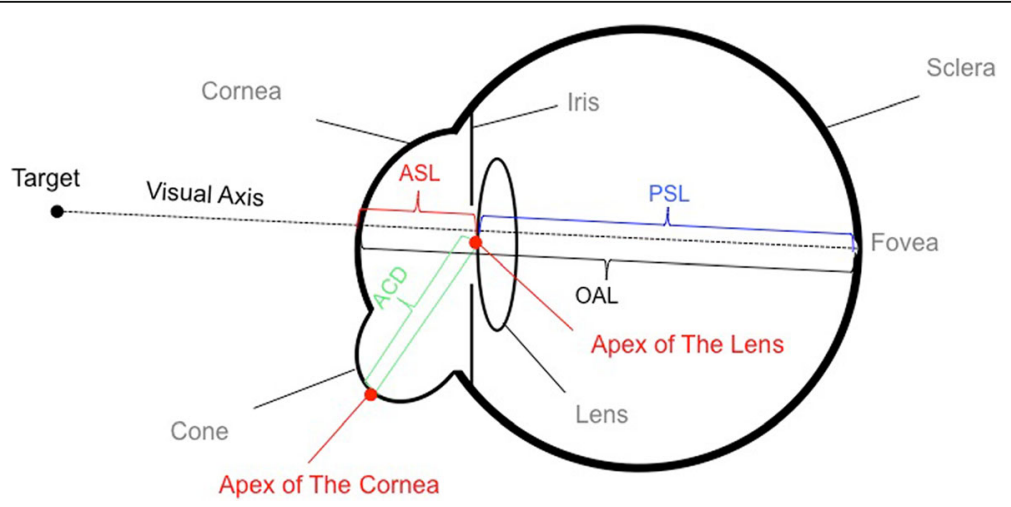

Fig. 1 The posterior segment length (PSL), which refers to the distance from the anterior capsule of the lens to the retinal pigment epithelium layer of the macula, was calculated with the formula "PSL =OAL-ASL" 
Table 1 Main Ocular Parameters $(n=106)$

\begin{tabular}{|c|c|c|c|c|c|c|c|}
\hline \multirow[t]{2}{*}{ Variables } & \multicolumn{2}{|c|}{ Worse Eyes $(n=53)$} & \multicolumn{2}{|c|}{ Fellow Eyes $(n=53)$} & \multirow[t]{2}{*}{$t$ value $^{a}$} & \multirow[t]{2}{*}{$P$ value } & \multirow{2}{*}{$\begin{array}{l}\text { D-value } \\
\text { Mean } \pm S D\end{array}$} \\
\hline & Mean \pm SD & Range & Mean \pm SD & Range & & & \\
\hline K1 (D) & $50.72 \pm 7.60$ & 41.40 to 70.70 & $43.77 \pm 2.95$ & 35.50 to 54.30 & 6.922 & $<0.001^{b}$ & $6.95 \pm 7.30$ \\
\hline K2 (D) & $55.56 \pm 8.15$ & 43.20 to 74.60 & $46.38 \pm 3.62$ & 41.30 to 56.10 & 8.614 & $<0.001^{\mathrm{b}}$ & $9.18 \pm 7.75$ \\
\hline Kmax (D) & $63.79 \pm 11.49$ & 45.20 to 86.90 & $50.03 \pm 6.57$ & 42.10 to 69.00 & 9.791 & $<0.001^{\mathrm{b}}$ & $13.76 \pm 10.23$ \\
\hline $\mathrm{CCT}(\mu \mathrm{m})$ & $477.5 \pm 42.7$ & 377 to 576 & $515.0 \pm 37.5$ & 424 to 587 & -8.350 & $<0.001^{b}$ & $-37.57 \pm 32.75$ \\
\hline $\mathrm{TCT}(\mu \mathrm{m})$ & $459.3 \pm 45.8$ & 331 to 541 & $504.3 \pm 39.9$ & 404 to 583 & -7.933 & $<0.001^{b}$ & $-45 \pm 41.30$ \\
\hline $\mathrm{ACD}(\mathrm{mm})$ & $3.51 \pm 0.32$ & 2.62 to 4.39 & $3.36 \pm 0.29$ & 2.65 to 3.91 & 4.539 & $<0.001^{b}$ & $0.15 \pm 0.24$ \\
\hline $\mathrm{OAL}(\mathrm{mm})$ & $24.76 \pm 1.24$ & 22.26 to 27.28 & $24.97 \pm 1.40$ & 22.37 to 27.70 & -3.120 & $0.003^{b}$ & $-0.21 \pm 0.49$ \\
\hline ASL (mm) & $4.01 \pm 0.30$ & 3.19 to 4.88 & $3.94 \pm 0.35$ & 3.19 to 5.46 & 1.876 & 0.066 & $0.07 \pm 0.25$ \\
\hline PSL (mm) & $20.76 \pm 1.15$ & 18.58 to 23.26 & $21.03 \pm 1.31$ & 18.50 to 23.54 & -4.537 & $<0.001^{b}$ & $-0.27 \pm 0.44$ \\
\hline
\end{tabular}

$M R S E=$ manifest refraction spherical equivalent; $D=$ diopter; $K 1=$ flat keratometry value; $K 2=$ steep keratometry value; $K m a x=$ maximum keratometry value; $C C T=$ central corneal thickness; $T C T=$ thinnest corneal thickness, $A C D=$ anterior chamber depth; $O A L=$ optical axial length; $A S L=$ anterior segment length; $P S L=$ posterior segment length ${ }^{\text {apaird t-test }}$

${ }^{\text {b }}$ Significant difference was detected between groups

\section{Main ocular dimensional parameters}

The mean ACD, OAL, ASL, and PSL values of the Severe Group were $3.51 \pm 0.32 \mathrm{~mm}, 24.76 \pm 1.24 \mathrm{~mm}$, $4.01 \pm 0.30 \mathrm{~mm}$ and $20.76 \pm 1.15 \mathrm{~mm}$, respectively. While those of the Mild Group were $3.36 \pm 0.29 \mathrm{~mm}, 24.97 \pm$ $1.40 \mathrm{~mm}, 3.94 \pm 0.35 \mathrm{~mm}$ and $21.03 \pm 1.31 \mathrm{~mm}$, respectively. The mean ACD value of the Severe Group was significantly higher than the Mild Group $(\mathrm{t}=4.539, P<$ 0.001), but the mean OAL and PSL values of the Severe Group were remarkably lower, when compared with the fellow eyes $(\mathrm{t}=-3.120, P=0.003 ; \mathrm{t}=-4.537, P<0.001$, respectively). No statistically significant differences were detected $(t=1.876, P=0.066)$ in the mean ASL values between groups (Fig. 2).

Correlations between the ocular dimensional parameters For the Severe Group, as shown in the Figs. 3 and 4, the Kmax values were significantly correlated with the SE

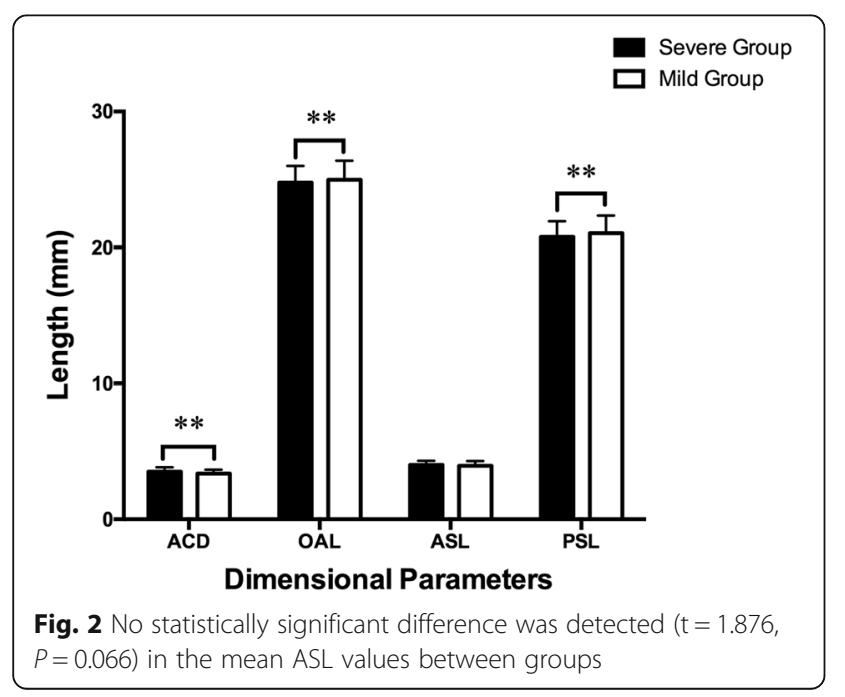

values $(\mathrm{R}=-0.385, P=0.004)$, the $A C D$ values $(\mathrm{R}=0.375$, $P=0.006)$, the ASL values $(\mathrm{R}=0.308, P=0.025)$ and the PSL values $(\mathrm{R}=-0.317, P=0.021)$, but not with the OAL values $(\mathrm{R}=-0.220, P=0.114)$. In the Mild Group, as demonstrated in the Figs. 3 and 5, the Kmax values were negatively correlated with the SE $(\mathrm{R}=-0.577, P<0.001)$, OAL $(\mathrm{R}=-0.533, \mathrm{P}<0.001)$, and PSL $(\mathrm{R}=-0.523, P<$ $0.001)$ values, but not with $\mathrm{ACD}(\mathrm{R}=-0.110, P=0.434)$ or ASL $(\mathrm{R}=-0.182, P=0.192)$ values.

\section{Discussion}

Keratoconus commonly leads to refractive error (myopia and irregular astigmatism) and visual impairment, which is very hard to fully correct using spectacles. Evidence has shown that, due to the ectasia of the cornea, adult keratoconic eyes are characterized by longer axial length and deeper anterior chamber depth, compared with normal eyes [23]. But our knowledge on the ocular dimensions of adolescent $\mathrm{KC}$ patients is still limited.

In the present study, we investigated corneal topographies and ocular dimensions of 53 adolescent $\mathrm{KC}$

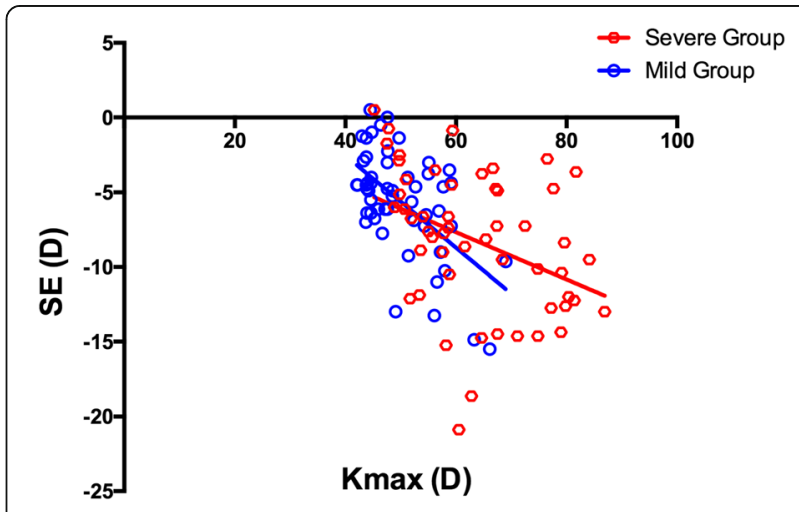

Fig. 3 The Kmax values significantly correlated with the SE values 


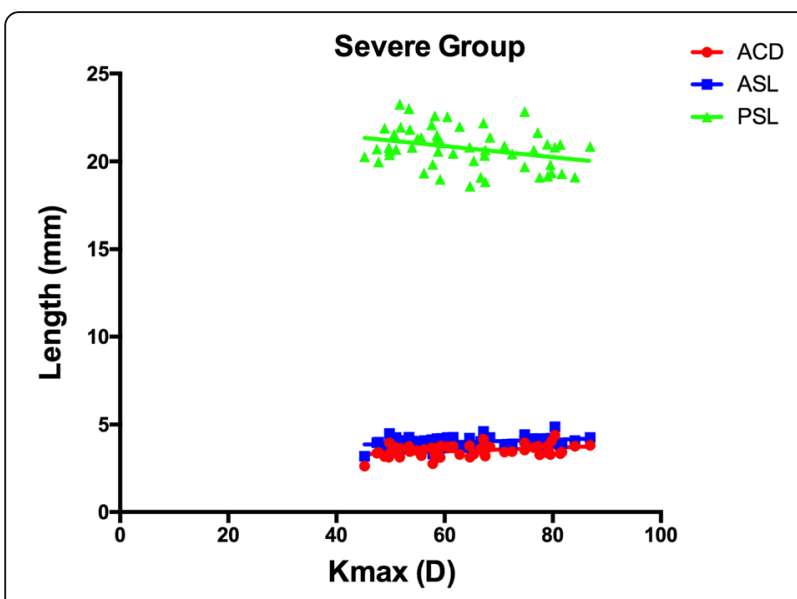

Fig. 4 In Severe Group, the Kmax values significantly correlated with the ASL values $(R=0.308, P=0.025)$ and the PSL values. $(R=-0.317, P=0.021)$

patients. Similar to adult $\mathrm{KC}$, the more severely affected eyes of these adolescent $\mathrm{KC}$ patients have steeper corneal curvature, thinner corneal thickness and higher myopia when compared with their less affected ones. Moreover, the ACD values of the more severely affected eyes are higher than their counterparts, while the difference in ASL values between the severe and fellow eyes is not significant. A possible explanation is that the Pentacam HR evaluates ASL along the visual axis, but measures ACD from the corneal apex (the steepest point of the anterior corneal surface). It is known that, for a young keratoconic eye, the steepest point of the cone (the corneal apex) is most frequently located in the paracentral or peripheral area of the cornea [24]. In contrast, the central cornea,

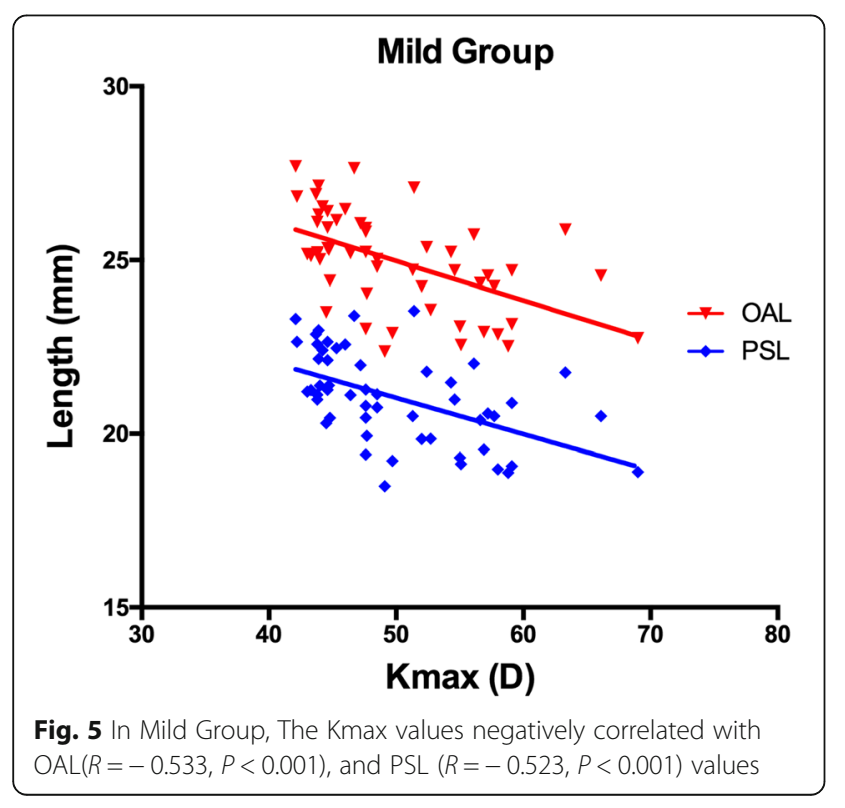

especially the visual axial area may be less affected compared with the corneal apex.

It is noticeable that the PSL and OAL values of the Severe Group were shorter than those of the Mild Group. Further analysis showed that, for the eyes in the Severe Group, the values of Kmax were positively correlated with the values of ACD (consists with the findings of Mas-Aixala E [25] and Safarzadeh M [26]) but negatively correlated with the values of PSL. This indicates that, in these severe cases, $\mathrm{KC}$ may affect both the dimensions of the anterior (positive correlation) and posterior (negative correlation) segments. We also noticed that Kmax did not correlate with OAL in the Severe Group. One possible reason is that the shortened PSL partially compensated the deepened ACD, which affected the overall changes of the OAL.

In the Mild Group, both the PSL and OAL values were negatively correlated with the Kmax value. This implies that for the mild cases, $\mathrm{KC}$ mostly affects the posterior, rather than the anterior segment of an eye.

It is interesting that the mean OAL and PSL values of the Severe Group were much lower than those of the Mild Group. We hypothesize that severe cases of KC may lead to more severe myopia and astigmatism, which may induce stronger myopic defocus signals when compared with less affected eyes. It has been reported that peripheral myopic defocus could thicken choroid and inhibit eye growth in birds [27], primates [28] and even in human beings [29] during their emmetropization. As the cone of a keratoconic cornea is mostly located in the peripheral area, the peripheral retina may acquire the myopic defocus signal, and then trigger the mechanism, thus leading to the shortening of the PSL and OAL among those adolescent $\mathrm{KC}$ patients.

In our study, it was found that $\mathrm{KC}$ not only affected the refractive status but also the development of the axial length in adolescents. Because the development of the eyeball is not still in adolescents, the axial length is constantly changing. It refers that $\mathrm{KC}$ may lead more complex influence in adolescents. Therefore, when we dealing with adolescent $\mathrm{KC}$ patients, the axial length is also an important examination besides refractive status and corneal topography.

One limitation of our study is that the IOL-master was unable to evaluate the choroid thickness. Further studies are essential to determine the effects of optical defocus caused by kerotoconus on choroid thickness. Another limitation is that although most of the $\mathrm{KC}$ adolescents in the present study were initially diagnosed and never underwent any optical corrections, some of them had already been treated by spectacles. As keratoconus progresses silently and occurs successively with both eyes, the exposure time and dose of optical defocus in the earlier-onset eye (the more severely affected eye) are unquestionably longer and greater than the later-onset one. 


\section{Conclusions}

In conclusion, for $\mathrm{KC}$ adolescents, the more affected keratoconic eyes may be characterized by deeper ACD but shorter OAL and PSL, when compared with the less affected ones.

\section{Abbreviations}

ACD: Anterior chamber depth; ASL: Anterior segment length; BSCDVA: Best spectacles corrected distance visual acuity; CCT: Central corneal thickness; KC: Keratoconus; Kmax: Maximum keratometry value; OAL: Optical axial length; PSL: Posterior segment length

\section{Acknowledgments}

Not applicable.

\section{Funding}

1. National Natural Science Foundation of China (Grant No.81570879) http://www.nsfc.gov.cn

2. National Natural Science Foundation of China for Young Scholars (Grant No.81600762) http://www.nsfc.gov.cn

\section{Availability of data and materials}

Available upon request from the co-first authors Weijun Jian and Yang Shen.

\section{Authors' contributions}

XZ, WJ, YS Study concept and design; WJ, YS, YC data collection; MT, WJ, YS analysis and interpretation of data; WJ, YS drafting of the manuscript; XZ critical revision of the manuscript; $X Z$ supervision. All authors read and approved the final manuscript.

\section{Ethics approval and consent to participate}

This study was approved by the ethics committee of the Eye and ENT Hospital, Fudan University, and was carried out with due regard to the tenets of the Declaration of Helsinki. After a detailed explanation of the study, written informed consent was obtained from all the participants' legal agents.

\section{Consent for publication}

Not applicable.

\section{Competing interests}

The authors declare that they have no competing interests

\section{Publisher's Note}

Springer Nature remains neutral with regard to jurisdictional claims in published maps and institutional affiliations.

Received: 9 October 2017 Accepted: 8 February 2018 Published online: 13 February 2018

\section{References}

1. Ucakhan OO, Bayraktutar BN, Saglik A. Pediatric corneal collagen crosslinking: long-term follow-up of visual, refractive, and topographic outcomes. Cornea. 2016:35:162-8.

2. Ortiz-Toquero S, Perez S, Rodriguez G, de Juan V, Mayo-Iscar A, Martin R. The influence of the refractive correction on the vision-related quality of life in keratoconus patients. Qual Life Res. 2016;25:1043-51.

3. Arbelaez MC, Versaci F, Vestri G, Barboni P, Savini G. Use of a support vector machine for keratoconus and subclinical keratoconus detection by topographic and tomographic data. Ophthalmology. 2012;119:2231-8.

4. Meyer JJ, Gokul A, Vellara HR, Prime Z, McGhee CN. Repeatability and agreement of Orbscan II, Pentacam HR, and Galilei tomography Systems in Corneas with keratoconus. Am J Ophthalmol. 2017:175:122-8.

5. Luz A, Lopes B, Hallahan KM, Valbon B, Fontes B, Schor P, et al. Discriminant value of custom ocular response analyzer waveform derivatives in Forme Fruste keratoconus. Am J Ophthalmol. 2016;164:14-21.

6. Ambrosio RJ, Valbon BF, Faria-Correia F, Ramos I, Luz A. Scheimpflug imaging for laser refractive surgery. Curr Opin Ophthalmol. 2013;24:310-20.
7. Lopes BT, Ramos IC, Dawson DG, Belin MW, Ambrosio RJ. Detection of Ectatic corneal diseases based on Pentacam. Z Med Phys. 2016;26:136-42.

8. Shetty R, Arora V, Jayadev C, Nuijts RM, Kumar M, Puttaiah NK, et al. Repeatability and agreement of three Scheimpflug-based imaging Systems for Measuring Anterior Segment Parameters in keratoconus. Invest Ophthalmol Vis Sci. 2014:55:5263-8.

9. Godefrooij DA, de Wit GA, Uiterwaal CS, Imhof SM, Wisse RP. Age-specific incidence and prevalence of keratoconus: a Nationwide registration study. Am J Ophthalmol. 2017:175:169-72.

10. Wang D, Chun RK, Liu M, Lee RP, Sun Y, Zhang T, et al. Optical defocus rapidly changes choroidal thickness in schoolchildren. PLoS One. 2016;11: e161535.

11. Yamaguchi T, Ohnuma K, Konomi K, Satake Y, Shimazaki J, Negishi K. Peripheral optical quality and myopia progression in children. Graefes Arch Clin Exp Ophthalmol. 2013:251:2451-61.

12. Read SA, Collins MJ, Sander BP. Human optical axial length and defocus. Invest Ophthalmol Vis Sci. 2010;51:6262-9.

13. Abdala-Figuerola A, Navas A, Ramirez-Miranda A, Lichtinger A, HernandezBogantes E, Olivo-Payne A, et al. Scheimpflug and optical coherence tomography analysis of posterior keratoconus. Cornea. 2016;35:1368-71.

14. Sahebjada S, Xie J, Chan E, Snibson G, Daniel M, Baird PN. Assessment of anterior segment parameters of keratoconus eyes in an Australian population. Optom Vis Sci. 2014;91:803-9.

15. Nilsson M, Miller W, Cervino A, Bergmanson JP, Brautaset RL. Evaluation of the anterior chamber angle in keratoconus and normal subjects. Cont Lens Anterior Eye. 2015:38:277-82.

16. Rozema JJ, Zakaria N, Ruiz HI, Jongenelen S, Tassignon MJ, Koppen C. How abnormal is the noncorneal biometry of Keratoconic eyes? Cornea. 2016;35: 860-5.

17. Sahebjada S, Amirul IF, Wickremasinghe S, Daniell M, Baird PN. Assessment of macular parameter changes in patients with keratoconus using optical coherence tomography. J Ophthalmol. 2015;2015:245953.

18. Wang $X$, Dong J, Wu Q. Evaluation of anterior segment parameters and possible influencing factors in normal subjects using a dual Scheimpflug analyzer. PLoS One. 2014:9:e97913.

19. Abolbashari F, Mohidin N, Ahmadi HS, Mohd AB, Retnasabapathy S. Anterior segment characteristics of keratoconus eyes in a sample of Asian population. Cont Lens Anterior Eye. 2013;36:191-5.

20. Kovacs I, Mihaltz K, Nemeth J, Nagy ZZ. Anterior chamber characteristics of keratoconus assessed by rotating Scheimpflug imaging. J Cataract Refract Surg. 2010;36:1101-6.

21. Edmonds CR, Wung SF, Pemberton B, Surrett S. Comparison of anterior chamber depth of normal and keratoconus eyes using Scheimpflug photography. Eye Contact Lens. 2009:35:120-2.

22. Emre S, Doganay S, Yologlu S. Evaluation of anterior segment parameters in Keratoconic eyes measured with the Pentacam system. J Cataract Refract Surg. 2007;33:1708-12

23. Ernst BJ, Hsu HY. Keratoconus association with axial myopia: a prospective biometric study. Eye Contact Lens. 2011;37:2-05.

24. Ertan A, Kamburoglu G, Colin J. Location of steepest corneal area of cone in keratoconus stratified by age using Pentacam. J Refract Surg. 2009;25:1012-6.

25. Mas-Aixala E, Gispets J, Lupon N, Cardona G. The variability of corneal and anterior segment parameters in keratoconus. Cont Lens Anterior Eye. 2016; 39:466-70.

26. Safarzadeh M, Nasiri N. Anterior segment characteristics in normal and keratoconus eyes evaluated with a combined Scheimpflug/Placido corneal imaging device. J Curr Ophthalmol. 2016:28:106-11.

27. Wallman J, Gottlieb MD, Rajaram V, Fugate-Wentzek LA. Local retinal regions control local eye growth and myopia. Science. 1987:237:73-7.

28. Smith ER, Hung LF, Huang J, Blasdel TL, Humbird TL, Bockhorst KH. Effects of optical defocus on refractive development in monkeys: evidence for local, regionally selective mechanisms. Invest Ophthalmol Vis Sci. 2010;51: 3864-73.

29. Berntsen DA, Barr CD, Mutti DO, Zadnik K. Peripheral defocus and myopia progression in myopic children randomly assigned to wear single vision and progressive addition lenses. Invest Ophthalmol Vis Sci. 2013;54:5761-70. 